\title{
Costo comparativo entre abordaje abierto y laparoscópico en cirugía resectiva de colon*
}

\author{
Drs. GONZALO URREJOLA S. ${ }^{1}$, PILAR GONZÁLEZ G. ${ }^{2}$, \\ JUAN STAMBUK M. ${ }^{1}$, CRISTIAN TALADRIZ R. ${ }^{1}$ \\ 1 Servicio de Cirugía. \\ 2 Departamento de Personal. \\ Hospital de la Fuerza Aérea de Chile, Santiago, Chile.
}

\begin{abstract}
Comparative costs of laparoscopic and open colorectal surgery

Background: In Chile, laparoscopic surgery is not universally used for colorectal diseases due to its learning curve and high costs. However this last drawback may not be true if all hospitalization costs are considered. Aim: To compare the costs of open and laparoscopic colonic excision surgery. Material and Methods: Data for all elective colectomies performed during 2005 and 2006 were retrieved from hospital records. Procedures were classified as open or laparoscopic and the total costs associated to the hospital stay of each group of procedures was obtained from the accounting office of the hospital. Results: Twenty nine patients aged 31 to 88 years $(16$ males), were subjected to laparoscopic surgery and 39 , aged 38 to 87 years (20 females), to open surgery. Seventy six percent of patients in the group of laparoscopic surgery were subjected to a left hemicolectomy and $54 \%$ of those in the group of open surgery, to a right hemicolectomy. Operative times were $188 \pm 14$ and $174 \pm 44$ min for open and laparoscopic surgery, respectively (NS). The item with higher costs for both groups was consumables, followed by hospitalization days. No differences in the total hospitalization costs were observed between the two groups of patients. Conclusions: Laparoscopic colorectal surgery does not have a higher economical cost than traditional open surgery.
\end{abstract}

Key words: Diverticulitis, diverticular disease, laparoscopy.

\section{Resumen}

Introducción: La cirugía laparoscópica ha tenido gran auge en los últimos años. Disminuye el trauma quirúrgico, minimizando la activación inmunológica y el catabolismo. Sin embargo, en Chile no es utilizada universalmente en patología colorectal por dos motivos: la curva de aprendizaje y el costo elevado que representa para el centro hospitalario o el paciente. Nuestra hipótesis es que la cirugía laparoscópica colorectal no es un evento más caro que la cirugía abierta, si se toma en cuenta toda la hospitalización. El objetivo de este trabajo es comparar el costo de la cirugía resectiva de colon laparoscópica y abierta, durante los años 2005-2006 en nuestro hospital. Métodos: De los registros del hospital y de la base de datos de cirugía colorectal se obtuvieron los datos de todas las colectomías electivas realizadas desde

*Recibido el 28 de Mayo de 2009 y aceptado para publicación el 13 de Julio de 2009.

Correspondencia: Dr. Gonzalo Urrejola S.

Dario Urzúa 2086, Depto 1304, Santiago, Chile.

E-mail: urregon@mac.com 
enero del 2005 hasta diciembre del 2006. Se constituyeron dos grupos; pacientes sometidos a cirugía laparoscópica y pacientes sometidos a cirugía tradicional abierta. Los datos económicos con respecto a cada evento, definido como colectomía electiva y su respectiva hospitalización, fueron obtenidos del registro de finanzas del hospital. Se analizó el costo comparativo con el software EPI-INFO. Resultados: Se estudiaron 68 casos operados. El procedimiento más frecuente en el grupo de cirugía laparoscópica fue la hemicolectomía izq (76\%), mientras que en el grupo de cirugía abierta fue la hemicolectomía derecha (54\%). No se encontró diferencia en los tiempos operatorios (188 \pm 41 minutos en cirugía abierta y $174 \pm 44$ minutos en cirugía laparoscópica). Para ambos grupos, el ítem más caro es el de insumos, seguido por los días-cama. No se encontró diferencias en las cuentas totales de ambos grupos. Discusión: Los resultados presentados muestran que la cirugía laparoscópica no tiene mayor costo que la cirugía tradicional abierta. La cirugía laparoscópica es el gold-estándar en la cirugía colónica. Este estudio parece demostrar que no existe un fundamento económico para no realizar de manera laparoscópica la cirugía de colon en nuestro centro.

Palabras clave: Costo, colorectal, laparoscopia, comparativo, colectomía.

\section{Introducción}

La cirugía mínimamente invasiva ha tenido gran aceptación en el medio quirúrgico actual. Los procedimientos laparoscópicos disminuyen el trauma quirúrgico, y de esta manera minimizan la activación inmunológica y el catabolismo como respuesta a la cirugía ${ }^{1,2}$.

En el campo de la cirugía colorectal, la resección intestinal laparoscópica ha sido empleada con éxito para el tratamiento de pólipos, enfermedad diverticular, prolapso rectal y enfermedad inflamatoria. La posibilidad de que esta vía de abordaje pudiera favorecer a pacientes intervenidos por cáncer colorectal fue planteada por primera vez en $1990^{3}$, sin embargo, la seguridad de este procedimiento ha sido cuestionada por trabajos iniciales que mostraban una recurrencia tumoral en las heridas de inserción de los trócares en el rango de $1,5 \%$ a $21 \% \%^{4,5}$.

Actualmente, gracias a los resultados de contundentes trabajos prospectivos randomizados multicéntricos, los problemas teóricos de resecciones oncológicas por vía laparoscópica han sido desmitificados $^{6,7}$. Se han asentado los beneficios, como reducción en dolor postoperatorio y uso de narcóticos ${ }^{8}$, disminución del íleo y las complicaciones post-quirúrgicas ${ }^{9}$, y menor tiempo de estadía hospitalaria y convalecencia ${ }^{10}$. La sobrevida y recurrencia presentada en la literatura es por lo menos igual a la de la cirugía tradicional, y la recurrencia en las heridas de trócares, que ahora se sabe que es multifactorial, ha disminuido a cifras cercanas al $1 \%{ }^{11}$, tasa similar a la reportada para cirugía colorectal abierta ${ }^{12}$.

Sin embargo, la aceptación de la técnica aún no es universal, por lo menos en nuestro país. Se plantea que lo anterior se debe en gran medida a dos razones fundamentales: la experiencia y curva de aprendizaje que se requiere en el procedimiento laparoscópico, y por otro lado, el costo involucrado en insumos y tiempo de uso de pabellón.

Si bien el tiempo operatorio y costo de pabellón publicados para esta cirugía son mayores que los de la cirugía tradicional, esto se compensa por el menor tiempo de estadía hospitalaria y otros costos asociados a un abordaje abierto (laboratorio, medicamentos, quinesiterapia, etc $)^{13}$.

En nuestro centro, a partir del año 2004 la cirugía laparoscópica de colon ha tenido progresiva aceptación, inicialmente en patología benigna, pero ahora empleándola en la mayoría de los pacientes, sean estos oncológicos o no. El costo que esto le ha significado al hospital no está estudiado.

Presentamos los datos acerca del costo de la cirugía resectiva de colon en el hospital $\mathrm{FACH}$, de los años 2005-2006, comparando la cirugía laparoscópica con el abordaje tradicional abierto. Nuestro objetivo es determinar si la técnica laparoscópica para la cirugía resectiva de colon es una alternativa económicamente viable, y comparar su costo con la cirugía abierta llevada a cabo en el mismo período de tiempo.

\section{Material y Método}

Se estudiaron los registros clínicos del hospital y la base de datos de cirugía colorectal, desde el $1^{\circ}$ de enero de 2005 hasta el 31 de diciembre de 2006, obteniendo los datos de todas las colectomías electivas. Se excluyó del análisis a las cirugías que tuvieron un procedimiento concomitante codificado asociado (colecistectomía, histerectomía, resección de metástasis hepáticas, etc).

Los datos económicos fueron obtenidos de los registros del departamento de finanzas del hospi- 
tal, analizando las planillas de cobro para cada caso individual, considerando el costo de la hospitalización y del evento quirúrgico, según los ítems expuestos en la Tabla 1. El análisis estadístico se realizó con el programa Epi Info, aplicando el test de ANOVA o Mann-Whitney/Wilconxon según correspondiera para el análisis de las medias. Se considera como estadísticamente significativo un valor $p<0,05$. El análisis se hizo según intención a tratar.

El número total de cirugías estudiadas fue de 68. Las características demográficas de los pacientes se exponen en la Tabla 2.

El diagnóstico más frecuente en el grupo de cirugía laparoscópica fue enfermedad diverticular (41\%), en cambio en cirugía abierta fue cáncer de colon $(79 \%)$.

El procedimiento más frecuente en el grupo de

Tabla 1. Datos analizados a partir de los registros económicos del hospital, para cada evento (definido como colectomía electiva y su respectiva hospitalización)

\begin{tabular}{l}
\hline Datos económicos analizados por paciente \\
\hline Días cama \\
Procedimiento quirúrgico \\
Medicamentos \\
Exámenes de laboratorio \\
Imagenología \\
Rehabilitación y kinesiterapia \\
Insumos \\
Cuenta total \\
\hline
\end{tabular}

cirugía laparoscópica fue la hemicolectomía izquierda $(\mathrm{n}=22,76 \%)$, en el grupo abierto fue la hemicolectomía derecha $(n=21,54 \%)$.

\section{Resultados}

No hubo diferencias en la duración de la cirugía, en cambio la estadía hospitalaria fue mayor en el grupo de cirugía abierta (Tabla 3).

El ítem de mayor costo en ambos tipos de procedimientos fue el de insumos, seguido por días-cama. En ambos grupos, los exámenes de imágenes y la medicina física y kinesiterapia dieron cuenta del menor gasto.

No se encontró diferencia entre las cuentas totales de ambos grupos. Los insumos fueron más caros en el grupo laparoscópico. Hubo diferencia a favor de cirugía laparoscópica en cuanto a díascama, medicamentos, exámenes de laboratorio, imagenología y rehabilitación. El costo de pabellón no presentó diferencia entre ambos grupos (Tabla 4).

\section{Discusión}

Parece claro que la cirugía laparoscópica colorectal presenta beneficios, por lo menos a corto plazo, con respecto a los procedimientos tradicionales abiertos. Esta cirugía se asocia a incisiones más pequeñas, menor dolor, y acelerada recuperación y convalecencia. En cuanto a las desventajas, la mayoría de los estudios han mostrado tiempos operatorios significativamente más largos con la cirugía laparoscópica comparados con la técnica tradicional $^{14,15}$, manteniéndose una diferencia cercana a 30 minutos.

Los estudios de análisis económico publicados,

Tabla 2. Características demográficas de los pacientes

\begin{tabular}{|c|c|c|}
\hline Característica & Colectomía laparoscópica $(n=29)$ & Colectomía abierta $(n=39)$ \\
\hline Edad promedio (rango) & $58(31-88)$ & $67(38-87)$ \\
\hline Hombres & 16 & 20 \\
\hline Mujeres & 13 & 19 \\
\hline ASA I y II, n (\%) & $25 \quad(86)$ & $(85)$ \\
\hline ASA III y IV, n (\%) & (14) & $(15)$ \\
\hline Cáncer & 10 & 31 \\
\hline Enfermedad diverticular & 12 & 4 \\
\hline Otros* & 7 & 4 \\
\hline
\end{tabular}

*Otros: pólipos, constipación, megacolon, trauma. 
COSTO COMPARATIVO ENTRE ABORDAJE ABIERTO Y LAPAROSCÓPICO EN CIRUGÍA RESECTIVA DE COLON

Tabla 3. Tiempo operatorio y de estadía hospitalaria, expresado en promedio \pm desviación estándar

\begin{tabular}{lccc}
\hline Grupo & Colectomía abierta & Colectomía laparoscópica & Valor p \\
\hline Minutos & $194 \pm 54$ & $180 \pm 70$ & $\mathrm{~ns}$ \\
Hospitalización (días) & $12,4 \pm 6,1$ & $7,1 \pm 3,1$ & $<0,001$ \\
\hline
\end{tabular}

Tabla 4. Costo hospitalario por procedimiento. Valores expresados en pesos

\begin{tabular}{lccc}
\hline Costos hospitalarios & Colectomía abierta & Colectomía laparoscópica & Valor p \\
\hline Días cama & 416.828 & 185.853 & $<0,001$ \\
Costo pabellón & 233.058 & 228.928 & $\mathrm{~ns}$ \\
Medicamentos & 416.471 & 179.037 & 0,0012 \\
Exámenes de laboratorio & 112.588 & 37.993 & 0,028 \\
Imágenes & 53.734 & 8.459 & 0,004 \\
Medicina física y rehabilitación & 61.971 & 23.378 & 0,02 \\
Insumos & 611.971 & 1.275 .566 & $<0,001$ \\
Cuenta Total & 2.033 .584 & 2.027 .512 & $\mathrm{~ns}$ \\
\hline
\end{tabular}

coinciden en que la cirugía mínimamente invasiva presenta mayores costos operatorios, pero menor costo asociado a terapéutica post-quirúrgica y estadía hospitalaria ${ }^{16}$.

No obstante, no podemos dejar de considerar la carga económica asociada al mayor tiempo de convalecencia y/o reposo médico que presenta el abordaje tradicional. Esto es especialmente cierto en un centro institucional como el nuestro, donde finalmente es la misma entidad la encargada de costear la hospitalización y el período de inactividad laboral de la convalecencia.

Es en este concepto de costo postoperatorio extrahospitalario donde reside una de las mayores ventajas de la cirugía laparoscópica.

También es necesario mencionar el concepto de "costo-oportunidad" asociado a estos distintos abordajes. Al ser mayor el tiempo hospitalario en la cirugía tradicional, estamos negando oportunidad para usar estos recursos hospitalarios para la resolución de patología programada en espera.

En nuestro trabajo no encontramos diferencia en los costos hospitalarios totales entre los pacientes operados por vía laparoscópica y abierta. Aunque tiene el inconveniente de sesgo de selección, al no haber sido un estudio prospectivo aleatorio, creemos que aporta datos para plantear que la técnica laparoscópica para la cirugía resectiva de colon es una alternativa comparable del punto de vis- ta económico con el abordaje tradicional abierto, no presentando un mayor costo si se considera toda la hospitalización del paciente.

\section{Referencias}

1. Busch O, Hop W, Marquet R, Jeekel J. Prognostic impact of blood transfusions on disease-free survival in colorectal carcinoma. Scand J Gastroenterol Suppl 1993; 200: 21-23.

2. Allendorf J, Bessler M, Whelan R, Trokel M, Laird D, Terry M, et al. Postoperative immune function varies inversely with the degree of surgical trauma in a murine model. Surg Endosc 1997; 11: 427-430.

3. Phillips E, Franklin M, Carroll B, Fallas M, Ramos R, Rosenthal D. Laparoscopic colectomy. Ann Surg 1992; 216: 703-707.

4. Tomita H, Marcello P, Milsom J. Laparoscopic surgery of the colon and rectum. World J Surg 1999; 23: 397405.

5. Reilly W, Nelson H, Schroeder G, Wieland H, Bolton $\mathrm{J}$, O'Connell M. Wound recurrence following conventional treatment of colorectal cancer. A rare but perhaps underestimated problem. Dis Colon Rectum 1996; 39: 200-207.

6. The Clinical Outcomes of Surgical Therapy Study Group. A Comparison of Laparoscopically Assisted and Open Colectomy of Colon Cancer. N Engl J Med 
GONZALO URREJOLA S. y cols.

2004; 350: 2050-2059.

7. The Colon Cancer Laparoscopic or Open Resection Study Group. Laparoscopic surgery versus open surgery for colon cancer: short-term outcomes of a randomised trial. Lancet Oncol 2005; 6: 477-484.

8. Milsom J, Bohm B, Hammerhofer K, Fazio V, Steiger $\mathrm{E}$, Elson P. A prospective, randomized trial comparing laparoscopic versus conventional techniques in colorectal cancer surgery: a preliminary report. J Am Coll Surg 1998; 187: 46-54.

9. Lacy A, García-Valdecasas J, Delgado S, Castells A, Taurá P, Piqué J, et al. Laparoscopy-assisted colectomy versus open colectomy for treatment of non-metastatic colon cancer: a randomised trial. Lancet 2002: 359: 2224-2229.

10. Franklin M, Rosenthal D, Abrego-Medina D, Dorman J, Glass J, Norem R, et al. Prospective comparison of open $v s$ laparoscopic colon surgery for carcinoma. Fiveyear results. Dis Colon Rectum 1996; 39: S35-S46.

11. Milsom J, Hammerhofer K. Role of laparoscopic techniques in colorectal cancer surgery. Oncology (Huntingt) 1995; 9: 393-398.

12. Talac R, Nelson H. Laparoscopic colon and rectal surgery. Surg Oncol Clin North Am 2000; 9: 1-12.

13. Delaney C, Kiran R, Senagore A, Brady K, Fazio V. Case-Matched Comparison of Clinical and Financial Outcome After Laparoscopic or Open Colorectal Surgery. Ann Surg 2003; 238: 67-72.

14. Milsom J, Bohm B, Hammerhofer K, Fazio V, Steiger $\mathrm{E}$, Elson P. A prospective, randomized trial comparing laparoscopic versus conventional techniques in colorectal cancer surgery: a preliminary report. J Am Coll Surg 1998; 187: 46-54.

15. Delgado S, Lacy A, García-Valdecasas J, Balaguí C, Pera M, Salvador L, et al. Could age be an indication for laparoscopic colectomy in colorectal cancer? Surg Endosc 2000; 14: 22-26.

16. Salloum R, Bulter D, Schwartz S. Economic Evaluation of Minimally Invasive Colectomy. J Am Coll Surg 2006; 202: 269-274. 